\title{
Supporting Information for: Detection of Acetylcholine at Nanoscale NPOE/Water Liquid/Liquid Interface Electrodes
}

\author{
Henry D. Jetmore \\ Department of Chemistry, The Beckman Institute for Advanced Science and Technology, University of Illinois at Urbana- \\ Champaign, Urbana, IL 61801, United States \\ †: Equal contribution \\ *Corresponding author \\ E-mail address: mshen233@illinois.edu
}

This supporting information contains the following:

Thermodynamics of ITIES: A simple case of ion transfer

Application of Least-Squares Regression to Determine $\alpha$ and $k^{0}$

Table S1: Simulation parameters for ion transfer across the NPOE/water nanointerface.

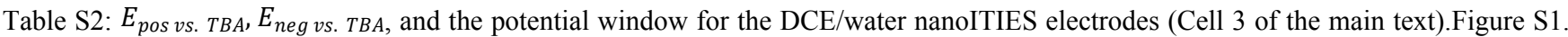
Linear calibration curve of acetylcholine detection using (A) cyclic voltammetry corresponding to Figure $3 \mathrm{~A}$ of the main text, (B) i-t amperometry corresponding to Figure 3B of the main text. (C) Background subtracted cyclic voltammograms of in vitro concentrationdependent acetylcholine (ACh) detection.

References 


\section{Thermodynamics of ITIES: A simple case of ion transfer}

Ions are detected by nanoITIES electrodes, generating electrochemical current (detection signal) due to the ions' transfer across the oil-water interface. The theory of the thermodynamics of ion transfer at an ITIES electrode is presented. ${ }^{1,2}$ An equilibrium is established between the water $(w)$ and oil $(o)$ phases for the ion $M^{z}$, where $z$ is the charge of the ion as shown:

$$
M^{z}(w) \rightleftharpoons M^{Z}(o)
$$

Under equilibrium conditions, the electrochemical potentials $(\bar{\mu})$ of $M^{z}$ in both phases are equal:

$$
\bar{\mu}_{M^{z}}^{w}=\bar{\mu}_{M^{z}}^{o}
$$

Expanding the electrochemical potential in each phase, $w$ and $o$, respectively, gives:

$$
\begin{aligned}
& \bar{\mu}_{M^{z}}^{w}=\mu_{M^{z}}^{0, w}+R T \ln \left(\alpha_{M^{z}}^{w}\right)+z F \phi^{w} \\
& \bar{\mu}_{M^{z}}^{o}=\mu_{M^{z}}^{0, o}+R T \ln \left(\alpha_{M^{z}}^{o}\right)+z F \phi^{o}
\end{aligned}
$$

In Equations S3 and S4, the terms are the standard chemical potential of ion $M^{z}$ in phase $i\left(\mu_{M^{2}}^{0, i}\right)$, activity of ion $M^{z}$ in phase $i\left(\alpha_{M^{z}}^{i}\right)$, inner potential or Galvani potential of phase $i\left(\phi^{i}\right)$, the ideal gas constant $(R)$, temperature $(T)$, the charge on ion $M(z)$, and Faraday's constant $(F)$. The Galvani potential difference for the transfer of ion $M^{Z}$ from phase $w$ to phase $O$ can be expressed as:

$$
\Delta_{w}^{o} \phi=\phi^{o}-\phi^{w}
$$

Rearranging Equations S3 and S4, substituting them into Equation S2, rearranging them to equal the right side of Equation S5, and making another substitution into Equation S5 gives:

$$
\begin{gathered}
\Delta_{w}^{o} \phi=\left(\mu_{M^{Z}}^{0, w}-\mu_{M^{z}}^{0, o}\right) / z F+(R T / z F) \ln \left(\alpha_{M^{Z}}^{w} / \alpha_{M^{z}}^{o}\right) \\
\Delta_{w}^{o} \phi=\Delta_{w}^{o} \phi_{M^{z}}^{0}+(R T / z F) \ln \left({ }^{\alpha_{M^{z}}^{w}} / \alpha_{M^{z}}^{o}\right)
\end{gathered}
$$

where $\Delta_{w}^{o} \phi_{M^{z}}^{0}$ is the standard Galvani potential difference of transfer of ion $M^{z}$ from $w$ to $o$, which is:

$$
\Delta_{w}^{o} \phi_{M^{z}}^{0}=\left(\mu_{M^{z}}^{0, w}-\mu_{M^{z}}^{0, o}\right) / z F=\Delta G_{t r}^{0, w \rightarrow o} / z F
$$

where $\Delta G_{t r}^{0, w \rightarrow o}$ is the standard free energy required to transfer ion $M^{z}$ from $w$ to $o$. Because $\Delta_{w}^{o} \phi_{M^{z}}^{0}$ is specific to the ionic analyte being transferred and the phases that analyte is being transferred between, qualitative differentiation of analytes is possible using nanoITIES electrodes. 


\section{Application of Least-Squares Regression to Determine $\alpha$ and $\boldsymbol{k}^{0}$}

The theoretical $i$ for each voltammogram potential was compared with the experimental $i$ at the same potential to create a metric for how well the theoretical curve fit the experimental curve. This metric $(\Delta i)$ was the sum of the squares of the differences between the theoretical currents $\left(i_{\text {theoretical }} m\right)$ and experimental currents $\left(i_{\text {experimental, }} m\right)$. Mathematically this is represented as:

$$
\Delta i=\sum_{j}\left(i_{\text {theoretical }, j}-i_{\text {experimental }, j}\right)^{2}
$$

The range $j$ encompasses the range within the forward wave from the diffusion-limited region of egress of $\mathrm{TBA}^{+}$to the diffusionlimited region of ingress of TBA ${ }^{+}$. To find the values of $\alpha$ and $k^{0}$ that minimized $\Delta i$, Microsoft Excel's Solver function add-in was used to determine the minimum value of $\Delta i$ by modifying $\alpha$ and $k^{0}$ with the evolutionary solver method. When using the Solver function, $\alpha$ was constrained between 1 and 0 and $k^{0}$ was constrained between 10 and 0 . The determined values of $\alpha$ and $k^{0}$ were then rounded to 2 significant figures. To find an approximate minimum of $\Delta i$, surrounding values of $\alpha$ and $k^{0}$ were tested to determine the range over which a good fit was maintained. 
Table S1. Simulation parameters for ion transfer across the NPOE/water nanointerface.

\begin{tabular}{cll}
\hline Parameter & Value & Definition \\
\hline$Z$ & $96,485 \mathrm{C} / \mathrm{mol}$ & Faraday's constant \\
& 1 & TBA $^{+}$charge \\
$T$ & $8.314 \mathrm{~J} / \mathrm{mol} \mathrm{K}$ & Ideal gas constant \\
$D_{w}$ & $298 \mathrm{~K}$ & Temperature \\
$x$ & $5.1 \times 10^{-6} \mathrm{~cm}^{2} / \mathrm{s}^{3}$ & Diffusion coefficient of TBA ${ }^{+}$in water \\
$c_{w}$ & 1.23 & Parameter related to pipette geometry \\
$c_{N P O E}$ & $2 \mathrm{mM}$ & Concentration of TBA $^{+}$in water \\
\hline
\end{tabular}

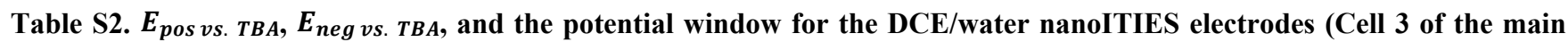
text).

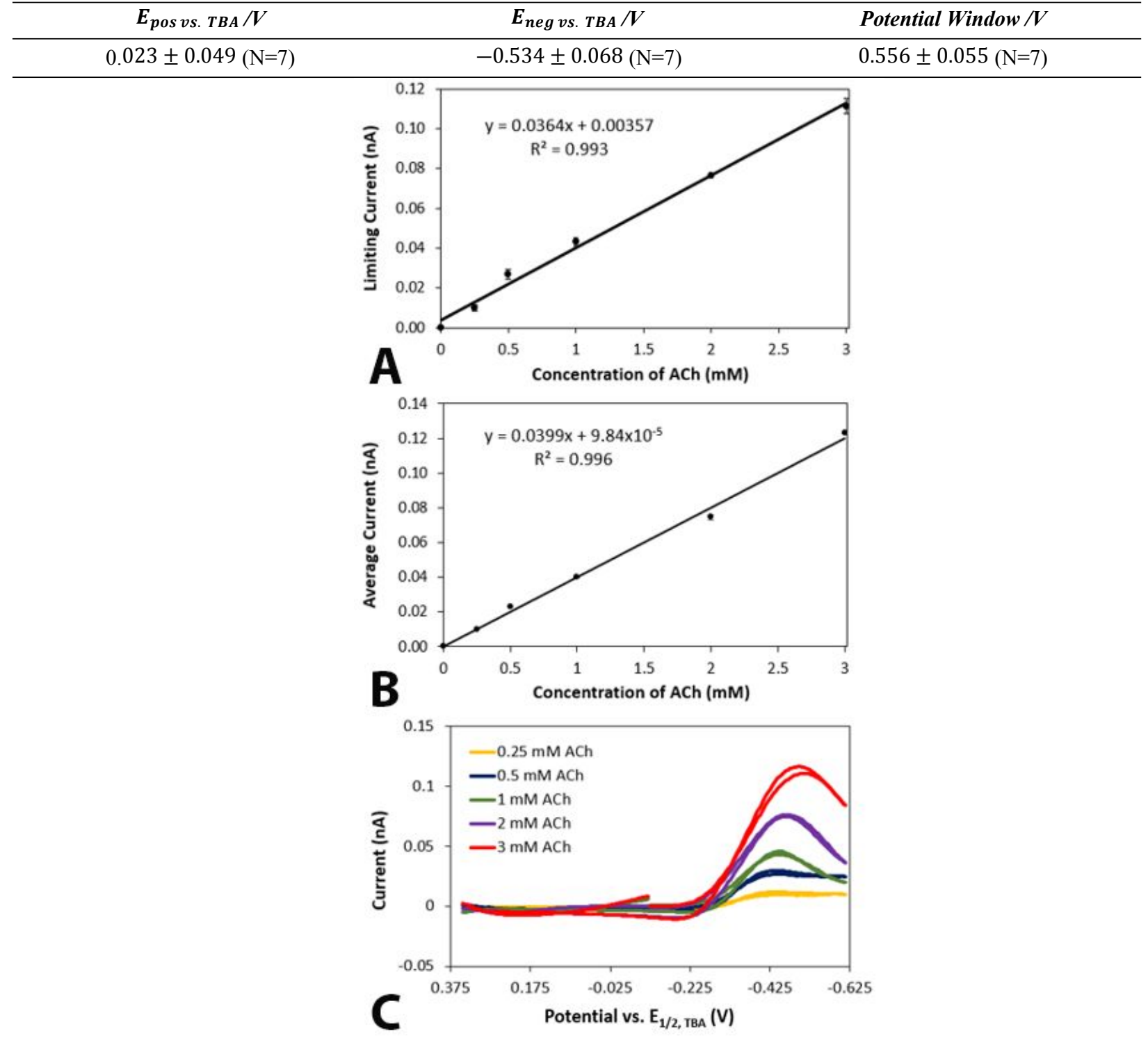

Figure S1. Linear calibration curve of acetylcholine detection using (A) cyclic voltammetry corresponding to Figure 3A of the main text, (B) i-t amperometry corresponding to Figure 3B of the main text. (C) Background subtracted cyclic voltammograms of in vitro concentrationdependent acetylcholine (ACh) detection. 


\section{References:}

1. Bard, A. J.; Faulkner, L. R., Electrochemical Methods Fundamental and Applications, 2nd Edition. 2001.

2. Bard, A. J.; Zoski, C. G., Electroanalytical Chemistry: A Series of Advances: Volume 23. CRC press: 2010 .

3. Shen, M.; Ishimatsu, R.; Kim, J.; Amemiya, S., Journal of the American Chemical Society 2012, 134 (24), 9856-9859. 